Meta

Journal des traducteurs

Translators' Journal

\title{
La traduction dans les agences de publicité
}

\section{Philippe Émond}

Volume 21, numéro 1, mars 1976

La traduction et l'entreprise

URI : https://id.erudit.org/iderudit/002708ar

DOI : https://doi.org/10.7202/002708ar

Aller au sommaire du numéro

Éditeur(s)

Les Presses de l'Université de Montréal

ISSN

0026-0452 (imprimé)

1492-1421 (numérique)

Découvrir la revue

Citer cet article

Émond, P. (1976). La traduction dans les agences de publicité. Meta, 21(1),

81-86. https://doi.org/10.7202/002708ar d'utilisation que vous pouvez consulter en ligne.

https://apropos.erudit.org/fr/usagers/politique-dutilisation/ 


\section{La traduction dans les agences de publicité}

\section{Que sont les agences de publicité?}

Avant de traiter des problèmes propres à la traduction dans les agences de publicité, il importe de définir en quoi consiste ces agences ainsi que leur mode de fonctionnement. En oubliant les cas d'exception que représentent de nombreuses petites ou moyennes agences très spécialisées, nous pouvons les classer arbitrairement en trois catégories :

a) Les agences commerciales : cette catégorie regroupe toutes les agences dont la publicité s'adresse directement aux consommateurs, c'est-à-dire le grand public, celui qui forme le plus important pouvoir d'achat. Responsable de la réclame pour tous les biens de consommation, de l'automobile à la bière en passant par les détersifs, les cosmétiques et les aliments préparés, ce groupe rassemble les plus grosses agences qui se partagent, s'arrachent et se reprennent des contrats de publicité de plusieurs millions. Elles représentent la principale source d'influence sur le langage populaire, par l'ampleur de la diffusion de leurs messages.

b) Les agences industrielles : les agences dites industrielles exercent leur activité dans des secteurs spécifiques. La publicité qu'elles pratiquent, moins rémunératrice parce que dirigée vers un auditoire plus restreint, est plus technique et moins spectaculaire que la première. Elle vise un public averti, donc moins crédule et moins vulnérable que l'ensemble des consommateurs, et se doit d'utiliser des moyens de diffusion différents. Ces agences sont de loin celles qui font la plus grande consommation de traduction, bien qu'il faille retenir qu'en publicité, traduction est presque toujours synonyme d'adaptation ou de reformulation.

c) Les agences promotionnelles : désigné ainsi faute de pouvoir mieux les identifier, ce groupe rassemble une infinité de petites agences dont l'action s'exerce généralement en marge des "mass media », à l'exception d'occasionnels recours aux quotidiens, aux hebdos régionaux et parfois à la télévision. Leur travail consiste entre autres à organiser l'inauguration et, par la suite, la promotion des centres commerciaux, de campagnes de motivation en milieux très spécialisés ainsi que de certaines activités de relations publiques. La diffusion restreinte de leur réclame, les budgets souvent très limités dont elles disposent obligent habituellement ces agences à se débrouiller avec les moyens du bord. La qualité de la langue est pour eux d'une importance très secondaire. Dans le cadre de cet article, elles représentent un groupe marginal que je choisis d'ignorer. 
Cette classification, établie strictement pour les besoins du présent article, n'existe pas comme telle dans le monde de la publicité. On y décline volontiers ses spécialités tout en refusant d'être catalogué, craignant que l'étiquette ne s'avère un aveu de limites que tous s'efforcent de dépasser.

Malgré la diversité de leurs structures et de leurs activités, les agences ont toutes un caractère commun que le linguiste, le terminologue ou le traducteur artisanal épris de bon français aura tendance à oublier. Toutes sont, sans exception, des entreprises commerciales dont le seul but est de réaliser des profits en moussant les ventes de leurs clients. L'aspect indirectement didactique de la publicité pourrait faire du traducteur ou rédacteur publicitaire un missionnaire du bon français. Mais l'apostolat se concilie très mal avec les affaires, et les gigantesques entreprises que sont les grandes maisons de publicité se soucient très peu de l'approbation de l'Académie. Seule compte la réussite financière. La traduction n'est pour elles qu'une technique parmi beaucoup d'autres. Il ne faudra donc pas s'étonner de voir des agences appartenant à des anglophones et administrées par eux mettre de plus en plus l'accent sur une publicité authentiquement québécoise, puisque les résultats en démontrent quotidiennement la rentabilité. Nous pouvons alors assister à des situations cocasses où une agence dont la direction anglophone avoue être formellement opposée aux revendications nationalistes des Québécois, produira des commerciaux axés sur l'emploi de ce même nationalisme parce qu'il se révèle efficace dans certains cas. S'il s'est avéré depuis longtemps que l'argent n'a pas d'odeur, nous découvrons maintenant au Québec qu'il n'a pas non plus de langue.

\section{L'importance de la traduction en publicité}

Voyons maintenant quelle place occupe la traduction dans le domaine de la publicité où le renouvellement des techniques est une question de réussite et de survie.

Devant la lente mais apparemment irréversible progression du français au Québec, les publicitaires ont rapidement compris que ce marché risquait d'échapper à ceux qui n'auraient pas la clairvoyance de s'adresser aux Québécois dans « leur langue ». Que ceux qui interprètent les guillemets comme un sous-entendu pour joual se détrompent. Parler aux Québécois dans leur langue implique la nécessité d'utiliser une réclame conçue directement en fonction de la mentalité québécoise, c'est-à-dire par des Québécois, pour des Québécois, que cela entraîne l'emploi du joual ou pas. Cette constatation amena la mise sur pied de services français autonomes dans la plupart des grandes agences du type commercial. Ces services ne traduisent pas. Ils créent directement en français. Si le client insiste pour imposer un concept graphique originalement conçu par et pour des anglophones, la version française devient une adaptation du texte initial plutôt qu'une traduction. J'ignore à qui nous devons la cigarette "ébleuissante », mais cette réclame démontre une créativité autrement plus féconde que le beautiful blue anglais. Il va de soi qu'au sein de ces agences, la présence de rédacteurs à l'imagination fertile est plus recherchée que celle de trađucteurs, ceux-ci n'étant alors embauchés que pour leur connaissance des deux langues et leurs aptitudes stylistiques. Le surplus de traduc- 
teurs ne pouvant être utilisé par le service français au moment de sa création a été regroupé sous une nouvelle raison sociale; il constitue une agence de traduction filiale de la maison-mère et qui jouit d'une totale liberté d'action. Quand, par exception, le service requiert une traduction, il la confie à l'agence de traduction comme à tout autre pigiste ou sous-traitant, celle-ci devant pour survivre, aller recruter une partie de sa clientèle à l'extérieur de la maison-mère.

La rentabilité de la publicité française au Québec est si évidente que certaines agences américaines ont fondé des filiales québécoises entièrement composées de personnel autochtone. Ces satellites québécois bénéficient dès leur fondation de la clientèle des filiales canadiennes des firmes traitant avec leur maison-mère au-delà de la frontière. Il ressort que dans les agences susmentionnées, l'avenir appartient aux rédacteurs plutôt qu'aux traducteurs.

Par contre, les industriels et les commerçants qui comptaient autrefois sur l'agence de publicité pour traduire les textes techniques nécessaires, soit pour la seule compréhension du produit à annoncer, soit pour la rédaction des textes publicitaires, ont désormais tendance à former leur propre service de traduction. Cette décision découle surtout de la constatation que la qualité de la traduction ne résulte pas uniquement de la compréhension littérale du texte original mais de la connaissance pratique de l'objet de ce texte. Les portes qui se ferment dans les agences de publicité s'ouvrent dorénavant dans plusieurs sociétés qui, par le passé, ne s'étaient jamais souciées de la traduction française.

La situation décrite jusqu'à maintenant s'applique surtout aux agences commerciales. Reste le secteur dit industriel qui fonctionne d'une façon sensiblement différente. La plupart des agences de cette catégorie sont spécialisées dans un domaine spécifique de l'industrie. Leur rayon d'action est plus limité et exclut généralement la télévision, la radio et les quotidiens. Les périodiques spécialisés desservant le secteur visé constituent leur champ de bataille. À cette publicité particulière s'ajoute habituellement la production de la littérature publicitaire qui englobe les catalogues, les tarifs, les notices de montage et d'entretien ainsi que les divers dépliants publicitaires. Alors que l'agence commerciale se doit de faire vendre un produit grâce à l'utilisation de la connaissance des motivations d'un acheteur plus ou moins averti, sans entrer dans les complexités techniques, le rôle de l'agence industrielle est de renseigner un public déjà informé. Sa publicité s'adresse à des médecins, à des ingénieurs ou autres spécialistes de l'industrie. La qualité du texte est d'une importance primordiale. N'ayant pas à se soucier du grand public, et la langue de travail au Québec, surtout aux échelons supérieurs, étant presque exclusivement anglaise, cette publicité ne se fait parfois qu'en anglais, le vendeur et la majorité de ses clients étant presque tous anglophones. Si la traduction s'avère indispensable, l'agence recourra alors soit aux textes fournis par son client, si celui-ci peut s'offrir un service de traduction, soit à un traducteur spécialisé qui collabore avec elle depuis longtemps et avec lequel une étroite communication se sera établie. Que l'agence ait recourt aux traducteurs maison ou à un pigiste, elle ne peut éviter de nombreux problèmes qui, à la longue, risquent d'aliéner ses relations avec son client. 


\section{Problèmes particuliers}

Ayant déjà établi que les agences commerciales créent directement dans la langue du public auquel elles s'adressent, il s'ensuit que les problèmes de traduction demeurent surtout le lot des agences industrielles. Que l'agence se charge elle-même de la traduction ou qu'elle s'adresse à un pigiste, il lui reste à faire accepter le texte traduit. Il va de soi que le client qui exige de son agence qu'elle traduise sa publicité en français est une maison anglophone qui considère son personnel incompétent pour s'acquitter d'un tel travail. Cependant, lorsque vient le temps d'approuver le texte soumis, la première secrétaire venue, quand ce n'est pas un comptable ou un camionneur qui porte un nom français, devient un critique valable aux yeux de l'unilingue anglais. Il en résulte parfois des compromis totalement aberrants.

Une autre des difficultés rencontrées est l'incompatibilité de mentalité entre les traducteurs rompus aux impératifs du langage publicitaire et les traducteurs maison qui sont généralement des spécialistes ayant sacrifié la stylistique au profit de la terminologie de leur domaine particulier. Sans parler de certains traducteurs maison qui, en dehors de leur spécialité, maîtrisent plus facilement l'anglais du fait qu'ils côtoient quotidiennement des collègues de bureau anglophones. Le rédacteur ou traducteur publicitaire plus versé dans l'utilisation d'un langage profane ou idiomatique se butera alors à l'intransigeance ou à l'ignorance du spécialiste plus préoccupé par l'orthodoxie technique que par le ton commercial qui saura vendre le produit. Alors que le rédacteur publicitaire cherche à toucher le point sensible de l'acheteur éventuel sans toutefois trahir la teneur technique du message, le traducteur maison travaille dans l'absolu d'une langue qui se doit d'être aussi précise et objective que les produits dont il traite. La subjectivité du premier et l'objectivité du second sont parfois difficilement conciliables. Le seul arbitre étant le client unilingue anglophone, il penchera généralement en faveur de l'opinion de son employé.

À maintes reprises, la situation se complique par la pauvreté des textes à traduire. Il ne serait pas exagéré de dire que la traduction se fait souvent à partir de l'américain plutôt que de l'anglais. La chose ne poserait pas de problème si la rédaction était claire et précise. Cependant, bon nombre de textes sont ambigus, approximatifs et confus lorsqu'ils ne tombent pas carrément dans le mumbo jumbo. La traduction devient alors un véritable déchiffrage qui ne peut que provoquer chez son auteur le sentiment d'avoir accouché d'un travail aussi médiocre que celui qu'il avait à interpréter, à moins qu'il ne s'impose une recherche qui l'amènerait à dépasser le seuil de rentabilité à respecter pour survivre.

Bien que les difficultés rencontrées en traduction publicitaire soient de natures diverses, elles peuvent se résumer à un point principal : l'ignorance du français de la part du client. Si le client est francophone, il sera souvent réfractaire à une traduction en français standard parce qu'elle comptera trop de mots qui ne figurent pas à son vocabulaire et qu'elle sera dépourvue des anglicismes auxquels il est habitué. Sans admettre sa propre ignorance, il prendra prétexte de celle de sa clientèle pour exiger une révision du texte en un français plus primaire. Si le client est anglophone, le résultat sera sensiblement le même pour les raisons mentionnées précédemment. 


\section{Considérations}

Dans la conjoncture socio-politique actuelle, la profession de traducteur offre de nombreuses possibilités au Québec. À tort ou à raison, l'adoption de la Loi 22 a convaincu bon nombre d'hommes d'affaires anglophones de la nécessité de tout traduire en français, de leur papier à lettre jusqu'aux avis dans les toilettes. Mais, jusqu'ici, cette bilinguisation (il n'est pas encore question de francisation) n'est que très superficielle, puisqu'elle ne touche qu'aux communications officielles et à la publicité. La langue de travail demeure toujours l'anglais, et que celui qui en doute aille demander à un mécanicien de la Beauce ou de la Mauricie de changer la bobine ou les plots de contact de son auto. La bilinguisation forcée par la Loi 22 n'est en somme qu'un pâle reflet de la prise de conscience par les Québécois de leur identité. La sensibilité des publicitaires à capter les sentiments qui motivent le public leur a permis de percevoir ce phénomène et de l'exploiter à l'avantage de leurs clients : «On est six millions presque parents... Cette évolution collective, qui semble actuellement irréversible, explique la rapidité avec laquelle les grandes agences commerciales ont établi des services entièrement français. Les agences industrielles qui n'ont pas encore ressenti la nécessité de se mettre au pas seront bientôt obligées de s'adapter aux nouvelles conditions. Leurs services français, qui bien souvent se résument à un francophone faisant office de correcteur des textes français soumis par leurs clients ou commandés à des pigistes, devront s'élargir pour faire place à des traducteurs et à des rédacteurs qualifiés. Cette perspective devient très rassurante pour la relève présentement en formation dans nos universités.

Les maisons d'enseignement ne peuvent cependant ignorer les transformations qu'imposera la francisation graduelle du Québec. Il a été démontré que tout un secteur de la publicité ne traduit plus. Il est logique de conclure que les agences industrielles devront tôt ou tard suivre la même voie. Leur type de publicité nécessitant une somme importante de traduction que le client n'est pas toujours en mesure de fournir, elles se devront de pouvoir leur offrir ce service. Là encore, l'avenir est plutôt prometteur pour les traducteurs. Par contre, la même logique nous incite à croire que la publicité au Québec sera un jour affaire de création et non plus de traduction. Il serait peut-être indiqué d'envisager dès maintenant un cours qui formerait des rédacteurs publicitaires bilingues.

Toutefois, qu'il s'agisse de traduction ou de rédaction publicitaire, le principal handicap à surmonter présentement est l'absence d'un style qui, tout en demeurant français, saurait exprimer la réalité nord-américaine qui a très peu en commun avec ce que la publicité française nous révèle. Les usages commerciaux qui existent en France ainsi que l'ensemble des produits offerts diffèrent à un tel point de ce que nous connaissons qu'il est souvent impossible de calquer le langage commercial français. Cette difficulté, tout en constituant encore pour les traducteurs un problème d'importance, deviendra à plus ou moins long terme un problème de terminologie qui ne saura trouver sa solution hors du Québec. Une conclusion s'impose. Dans le contexte de la francophonie, un style publicitaire québécois traduisant fidèlement la réalité nord-américaine ne pourrait être qu'idiomatique. 


\section{Conclusion}

En étudiant la situation précédemment décrite, il appert qu'un seul des deux principaux secteurs de la publicité offre encore des possibilités aux traducteurs. Puisque la disparition éventuelle de cette discipline du domaine qui nous intéresse semble chose admise, nous pourrions conclure que le sujet est clos. Ce jugement serait pour le moins hâtif. Il est téméraire d'anticiper sur les événements. Le cours de l'histoire ne se trace pas à l'avance. Il se constate après coup.

La promulgation du français comme seule langue de travail au Québec pourrait provoquer une avalanche de demandes de traduction qui affecterait probablement toutes les sphères où cette discipline est actuellement employée. Ce seul exemple m'incite à croire que l'avenir de la traduction en publicité aussi bien que dans les autres domaines est intimement lié à l'avenir politique du Québec. Chose certaine, un Québec français occuperait une armée de traducteurs traduisant du français à l'anglais.

Il apparaitra certainement paradoxal d'admettre, après ces quelques milliers de mots, que beaucoup de ce qui a été dit ne colle pas totalement à la réalité. Le tableau esquissé ne représente que la schématisation d'un monde extrêmement complexe. La vérité est que la publicité, comme le savent si bien tous ceux qui s'y adonnent, est le domaine de l'intangible. Chaque agence fonctionne d'une façon distincte. Chacune préconise des principes non seulement différents mais parfois diamétralement opposés. Ce labyrinthe de contradictions provient de la nécessité de continuellement proposer à la clientèle le principe nouveau qui accomplira les prodiges que la compétition n'a su réaliser. Tous sont à la recherche de la pierre philosophale qui éclipsera les concurrents par des innovations spectaculaires et des formules inédites qui se concrétiseront souvent par des néologismes ou des barbarismes retentissants. Cette incessante poursuite de l'originalité, compliquée par la nécessité d'exprimer les innovations techniques qui échappent au vocabulaire traditionnel, nous donnent des perles comme le « javellisant pour les non-javellisables ». L'apostolat des publicitaires risque d'engendrer des rejetons qui, à la longue, n'utiliseront que ce style publicitaire québécois réflétant la réalité changeante nordaméricaine. Le sacro-saint principe de l'usage-critère donnera-t-il raison aux publicitaires?

PhILIPPE Émond 